\title{
Segmented X-Ray Optics for Future Space Telescopes
}

\author{
Ryan S. McClelland \\ SGT Inc. \\ 7701 Greenbelt Road \\ Greenbelt, Maryland 20770 \\ 240-366-7776 \\ ryan.s.mcclelland@nasa.gov
}

\begin{abstract}
Lightweight and high resolution mirrors are needed for future space-based $\mathrm{X}$-ray telescopes to achieve advances in high-energy astrophysics. The slumped glass mirror technology in development at NASA GSFC aims to build X-ray mirror modules with an area to mass ratio of $\sim 17 \mathrm{~cm}^{2} / \mathrm{kg}$ at 1 $\mathrm{keV}$ and a resolution of 10 arc-sec Half Power Diameter (HPD) or better at an affordable cost. As the technology nears the performance requirements, additional engineering effort is needed to ensure the modules are compatible with space-flight. This paper describes Flight Mirror Assembly (FMA) designs for several X-ray astrophysics missions studied by NASA and defines generic driving requirements and subsequent verification tests necessary to advance technology readiness for mission implementation.
\end{abstract}

The requirement to perform $\mathrm{X}$-ray testing in a horizontal beam, based on the orientation of existing facilities, is particularly burdensome on the mirror technology, necessitating mechanical over-constraint of the mirror segments and stiffening of the modules in order to prevent selfweight deformation errors from dominating the measured performance. This requirement, in turn, drives the mass and complexity of the system while limiting the testable angular resolution. Design options for a vertical $X$-ray test facility alleviating these issues are explored. An alternate mirror and module design using kinematic constraint of the mirror segments, enabled by a vertical test facility, is proposed. The kinematic mounting concept has significant advantages including potential for higher angular resolution, simplified mirror integration, and relaxed thermal requirements. However, it presents new challenges including low vibration modes and imperfections in kinematic constraint. Implementation concepts overcoming these challenges are described along with preliminary test and analysis results demonstrating the feasibility of kinematically mounting slumped glass mirror segments.

\section{TABLE OF CONTENTS}

\section{INTRODUCTION}

\section{Progress on IXO Module Design AND}

ANALYSIS

\section{FUTURE DiRECTIONS IN MODULE AND FMA}

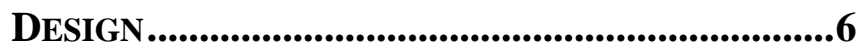

4. CONCLUSIONS ...................................................9

REFERENCES........................................................10

BIOGRAPHIES. .10 telescopes. While both lightweight and high resolution mirror fabrication technologies exist, no mature technology currently achieves both at once. For example, the Chandra mirror has a high resolution ( 0.5 arc-sec HPD) but is relatively heavy $\left(0.44 \mathrm{~cm}^{2} / \mathrm{kg} @ 1.0 \mathrm{keV}\right.$ effective area) while the Suzaku mirror has a low resolution (110 arc-sec HPD) but is very lightweight $\left(17.1 \mathrm{~cm}^{2} / \mathrm{kg} @ 1.0 \mathrm{keV}\right)[1]$. However, technology currently in development by the Next Generation X-Ray Optics (NGXO) team at NASA GSFC aims to achieve both high angular resolution (10 arc-sec HPD or better) and lightweight $\left(\sim 15 \mathrm{~cm}^{2} / \mathrm{kg} @ 1.0 \mathrm{keV}\right)$ mirrors at a cost consistent with future NASA mission budgets. This slumped glass mirror technology is scalable to a variety of $\mathrm{X}$-ray mission sizes and science objectives and has already been demonstrated to achieve $\sim 15$ arc-sec HPD performances in a full aperture X-ray test of multiple co-aligned mirror shells in a Technology Development Module [2]. Due to the recent progress by the NGXO team, detailed engineering of the modules that support the slumped glass segments is required to prepare the technology for mission implementation. We seek to mitigate the cost and schedule risks of implementing this technology through rigorous and repeated integration and testing of Technology Development Modules with increasing flight fidelity.

\section{Slumped Glass Mirror Technology Overview}

The mirror technology assumed for the module designs presented in this paper, currently in development at NASA GSFC, consists of $0.4 \mathrm{~mm}$ thick glass sheets slumped over polished convex mandrels [3]. The mirror segments thus formed are cut to size $(200 \mathrm{~mm}$ axial length and variable azimuthal span), coated with a thin layer of iridium, and temporarily mounted to a structure allowing for rigid body manipulation. Pairs of mirrors are then aligned to a common focus using rigid body manipulation, then permanently bonded into the module structure, and finally released from the temporary mount. Once the module is fully populated with mirror segments and the back of the module is closed out with a protective panel, as shown in Figure 1, the assembly is tested. Many modules are then coaligned into a common FMA structure to complete the Flight Mirror Assembly as shown in Figures 2 and 3.

\section{INTRODUCTION}

Advancements in X-ray optics fabrication technologies are required to enable future discoveries by space-based X-ray 


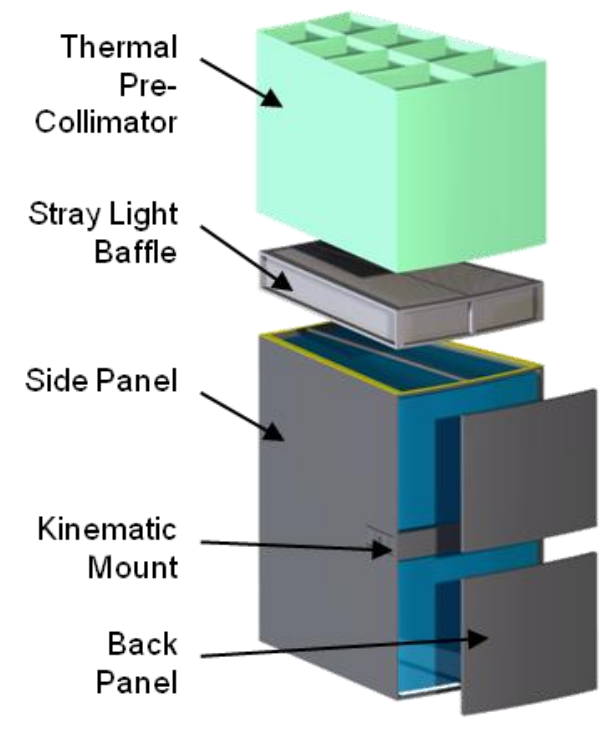

Figure 1. Exploded view of an individual mirror module

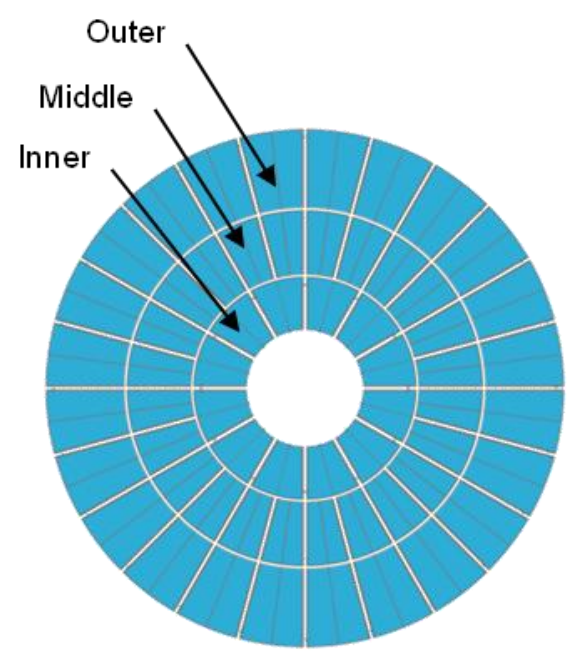

Figure 2. Top view of an FMA with three rings of modules illustrating the scalable modular approach

\section{Flight Mirror Assembly Overview}

The FMA combines tens of modules collectively containing thousands of mirror segments into a common structure with a common focus (though some missions studied have multiple foci). Significant FMA design and analysis was performed as part of the International X-Ray Observatory (IXO) mission study [4]. However, the design approach of combining rings of modules into a Carbon Fiber Reinforced Polymer (CFRP) super structure is scalable to a variety of mission sizes including Flagship Missions such as IXO, Missions of Opportunity, and Explorer Missions. The effective area at specific energies of an FMA design can be adjusted by changing the number and diameter of module rings. Figure 2 illustrates an FMA with 60 modules arranged in three rings while Figure 3 illustrates a 24 module FMA with two partially populated rings. Several FMA designs using the NGXO slumped glass mirror technology were studied as part of a broad X-ray mission study directed by NASA HQ [5]. Four mission concepts which would achieve significant portions of the IXO science goals were studied in detail at the GSFC Mission Design Lab (MDL). Key parameters for the FMA designs associated with each mission are shown in Table 2. Note that the Wide Field Imager (WFI) mission baselined a polished full shell mirror technology, however an alternate FMA design using the NGXO slumped glass technology is shown herein.

\section{Module REQUIREMENTS}

While challenging, creating the FMA from modules does not require any new technologies. The focus of the NGXO technology development effort lies in creating modules supporting hundreds of slumped glass mirror segments which have the required optical performance while also surviving the rigors of the launch and the on-orbit environments without significant performance degradation. Now that the NGXO team is near achieving the optical performance requirements, additional effort is needed to define the space-flight requirements, design and analyze Technology Development Modules to these requirements, and develop and implement a test plan verifying the requirements are met. Generic module requirements based on the study mission designs are summarized in Table 1 and each requirement is discussed in detail in the following sections. In every case, the requirements must be refined for mission specific module designs. The requirements listed here are not exhaustive, rather they are viewed as the requirements which drive technology development and module design.

Table 1. Overview of generic module requirements which drive design

\begin{tabular}{|l|c|c|}
\hline \multicolumn{1}{|c|}{ Requirement } & Value & Verification Test \\
\hline Angular Resolution & $8.6 \mathrm{arc}-\mathrm{sec} \mathrm{HPD}$ & X-ray Test \\
\hline Effective Area & $17 \mathrm{~cm}^{2} / \mathrm{kg} @ 1 \mathrm{keV}$ & X-ray Test \\
\hline $\begin{array}{l}\text { Quasi-static Design } \\
\text { Load }\end{array}$ & $9 \mathrm{~g}$ axial, $3 \mathrm{~g}$ lateral & $\begin{array}{c}\text { Sine Burst } \\
\text { Vibration Test }\end{array}$ \\
\hline Acoustic Load & $139.6 \mathrm{~dB}$ OASPL & Acoustic Test \\
\hline Random Vibration Load & GEVS Workmanship & $\begin{array}{c}\text { Random Vibration } \\
\text { Test }\end{array}$ \\
\hline Pyroshock Load & $3,000 \mathrm{~g}$ max & \begin{tabular}{c} 
Shock Test \\
\hline Survival Temperatures
\end{tabular} \\
\hline $0^{\circ}-30^{\circ} \mathrm{C}$ & $\begin{array}{c}\text { Thermal-Vacuum } \\
\text { Test }\end{array}$ \\
\hline Temperature Gradient & $0.1^{\circ} \mathrm{C}$ axial & $\begin{array}{c}\text { Thermal-Vacuum } \\
\text { X-ray Test }\end{array}$ \\
\hline $\begin{array}{l}\text { Bulk Temperature } \\
\text { change }\end{array}$ & $0.5^{\circ} \mathrm{C}$ & $\begin{array}{c}\text { Thermal-Vacuum } \\
\text { X-ray Test }\end{array}$ \\
\hline
\end{tabular}




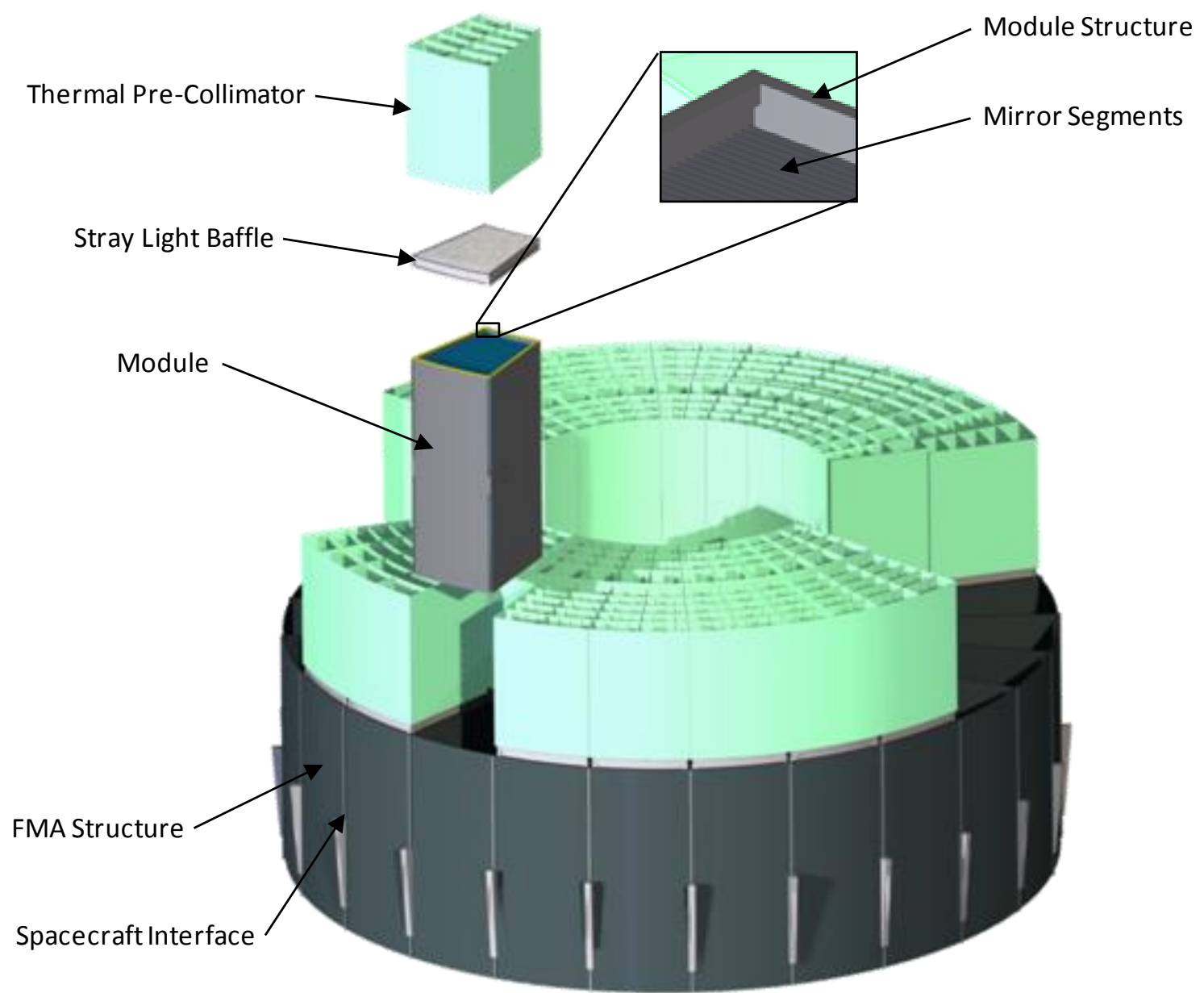

Figure 3. Exploded view of an FMA with two partially populated rings of modules

Table 2. Overview of FMA parameters for notional mission studied

\begin{tabular}{|c|c|c|c|c|c|c|}
\hline Study Mission & Instruments & $\begin{array}{c}\text { Effective Area } \\
\left(\mathrm{cm}^{2}\right)\end{array}$ & $\begin{array}{c}\text { FMA Mass } \\
(\mathrm{kg})\end{array}$ & $\begin{array}{c}\text { FMA Outer } \\
\text { Diameter }(\mathrm{m})\end{array}$ & $\begin{array}{c}\text { \# of } \\
\text { Modules }\end{array}$ & $\begin{array}{c}\text { \# of } \\
\text { Segments }\end{array}$ \\
\hline IXO [6] & $\begin{array}{l}\text { XMS, WFI, HWI, } \\
\text { XGS, HTRS, XPOL* }\end{array}$ & $\begin{array}{c}32,000 \\
@ 1.25 \mathrm{keV}\end{array}$ & 1750 & 3.0 & 60 & 13,896 \\
\hline AXSIO [8] & XMS, XGS & $\begin{array}{c}9,800 \\
@ 1 \mathrm{keV}\end{array}$ & 524 & 1.8 & 60 & 8,280 \\
\hline Notional WFI Mission & WFI & $\begin{array}{c}7,200 \\
\text { @ } 1 \mathrm{keV}\end{array}$ & 487 & 1.6 & 60 & 7,368 \\
\hline Notional Calorimeter Mission & XMS & $\begin{array}{c}5,000 \\
\text { @ } 1 \mathrm{keV}\end{array}$ & 319 & 1.3 & 36 & 5,856 \\
\hline Notional Gratings Mission & XGS & $\begin{array}{c}1,600 \\
@ 653 \mathrm{eV}\end{array}$ & 75 & 1.3 & 16 & 960 \\
\hline
\end{tabular}

\section{Optical Performance Requirements}

The optical performance requirements of the FMA are dependent on the scientific objectives of the mission. Effective areas at specific X-ray energy levels are particularly affected. However, some generic requirements at the module level can be developed. An angular resolution of 10 arc-sec HPD is sufficient to attain the scientific objectives of the notional missions listed in Table 2 . Assuming 5 arc-sec of error is reserved for integration and co-alignment of modules within the FMA, thermal distortion, and other telescope level effects, the modules must achieve a resolution of 8.6 arc-sec. While the effective area requirement is mission dependent, the effective area per unit mass can be generalized; $17 \mathrm{~cm} 2 / \mathrm{kg} @ 1 \mathrm{keV}$ is in line with requirements for the notional missions.

\section{Mechanical Load Requirements}

The modules experience significant mechanical loading environments during ground handling, transportation, 
launch, and verification testing. For the sake of design, analysis, and testing, the loads are generally divided into several categories [8]. For each category, a general description of the load is given, along with generic values for use in preliminary design. In every case, the loads must be refined on a mission specific basis.

\section{Quasi-static design loads}

Quasi-static design loads, typically expressed in factors of gravitational acceleration (g), represent the static, low frequency, and transient loads experience by the module. Initially, quasi-static design loads can be taken from the launch vehicle user's guide or from previous missions using the same launch vehicle. Once a preliminary design and Finite Element Model (FEM) of the spacecraft is created, a Coupled Loads Analysis (CLA), which simulates all phases of launch and accounts for the dynamics of the launch vehicle and spacecraft combined, is performed and high fidelity quasi-static loads are developed. CLA was performed for IXO assuming an Atlas 551 launch vehicle and module accelerations for each launch phase were recovered [9]. Maximum accelerations of $6.14 \mathrm{~g}$ axial and $1.69 \mathrm{~g}$ lateral occur during the maximum axial acceleration and transonic events respectively. These loads are nearly identical to the design loads specified in the Atlas V user's guide due to the stiff load path from the module to the launch vehicle interface and resulting low dynamic amplification. For preliminary design of a generic module, the launch vehicle design loads are multiplied by a 1.5 Model Uncertainly Factor (MUF) resulting in design loads of $9 \mathrm{~g}$ axial and $3 \mathrm{~g}$ lateral which envelope design loads for both Atlas V and Falcon 9, the likely launch vehicles for future X-ray missions. These loads are also likely to envelope any ground handling or transportation loads.

\section{Acoustic Loads}

Acoustic loads, typically expressed in $\mathrm{dB}$ over a $25-10,000$ $\mathrm{Hz}$ frequency range or simply as a Overall Sound Pressure Level (OASPL), represent the loads caused by sound pressure waves impinging on the spacecraft during launch. These loads can be taken directly from the launch vehicle user's guide. Acoustic loads of 139.6 dB OASPL envelope the likely launch vehicles.

\section{Random Vibration Loads}

Random vibration loads, typically expressed in $\mathrm{g}^{2} / \mathrm{Hz}$ over a $20-20,000 \mathrm{~Hz}$ frequency range, represent the loads transmitted from the structure to the module caused by acoustic loads on other parts of the spacecraft. Acoustic loads primarily affect lightweight structures with large areas such as solar arrays and metering structures. The modules, due to their protected location within the spacecraft and FMA structures [6] would primarily experience acoustic loads second-hand as structure borne random vibration loads. The random vibration spectrum is highly dependent on the spacecraft design; therefore the NASA GSFC General Environmental Verification Standard (GEVS)
Workmanship Random Vibration environment can be used for preliminary module design and testing.

\section{Pyrotechnic Shock Loads}

Pyrotechnic shock loads, typically expressed in factors of gravitational acceleration (g) over a $100-10,000 \mathrm{~Hz}$ frequency range, represent the loads caused by separation events during launch and on-orbit deployments. These loads are taken from the launch vehicle user's manual or from the specifications of selected release devices and attenuated by the load path between the shock initiation point and module. Current mission designs place the mirror modules near the launch vehicle interface which results in minimal shock attenuation. Shock transmission through a structure is difficult to model due to the high frequencies involved, therefore attenuation through the structure is first simply estimated based on the number of bolted joints and length of intervening structures then later determined accurately by spacecraft level testing. A maximum shock load of $3000 \mathrm{~g}$ at the spacecraft to launch vehicle interface envelopes the likely launch vehicles and separation systems, assuming a low shock clamp-band style separation system is used.

\section{Thermal and Vacuum Requirements}

The modules experience thermal loads, and therefore thermal distortions, during ground handling, storage, transportation, launch, and on-orbit operation. Thermal requirements can be broadly divided into operational temperature requirements and survival temperature requirements. Vacuum requirements are also addressed below.

\section{Operational Temperatures}

During performance testing on the ground and on-orbit science data collection, tight temperature control of the module must be maintained to ensure the angular resolution remains within requirements. Particularly challenging requirements must be levied upon slumped glass mirror modules due to the relatively high $6.3 \mathrm{ppm} /{ }^{\circ} \mathrm{C}$ Coefficient of Thermal Expansion (CTE) of the glass. Structural Thermal Optical Performance (STOP) analysis of mirror modules shows that gradients of only $0.1^{\circ} \mathrm{C}$ over a mirror segment or between the segment and structure can significantly degrade the performance [9]. Somewhat less stringent is the bulk temperature change requirement since the CTE of the glass can be well matched by structural materials. A bulk temperature change of $0.5^{\circ} \mathrm{C}$ can be tolerated and is readily achievable through heater control.

During testing and on-orbit the modules are also exposed to high vacuum. Materials, particularly adhesives must be selected and tested to have low out-gassing to prevent both figure distortion and mirror contamination.

\section{Survival Temperatures}

When the X-ray modules are not operating, such as in storage, cruising to final orbit, or during mission safe hold 
scenarios, a greater range of module temperatures is permitted. The modules must only survive these temperatures without unacceptable permanent deformation. However, these requirements can drive spacecraft and mission design by necessitating additional redundancy, limiting spacecraft orientation with respect to the sun, and requiring special storage facilities. The survival temperature requirements are initially determined by the temperature limits of the materials used to build the module and later based on testing. Of particular concern is creep of adhesives at elevated temperatures. Based on the Chandra mission, a preliminary requirement of $10^{\circ}-30^{\circ} \mathrm{C}$ is baselined though a requirement derived from thermal testing testing will be generated once the final adhesives have been selected.

\section{Verification Testing Program}

The generalized module requirements described here-in must be verified through testing in order to advance technology readiness in preparation for mission implementation. The goals is to mitigate the cost and schedules risks of implementing the NGXO mirror technology through rigorous and repeated integration and testing of Technology Development Modules. Table 1 enumerates the test associated with each requirement as well as the generic test levels. An X-ray performance test is completed before and after each environmental test in order to verify performance has not unacceptably degraded. A preliminary test flow is shown in Figure 4.

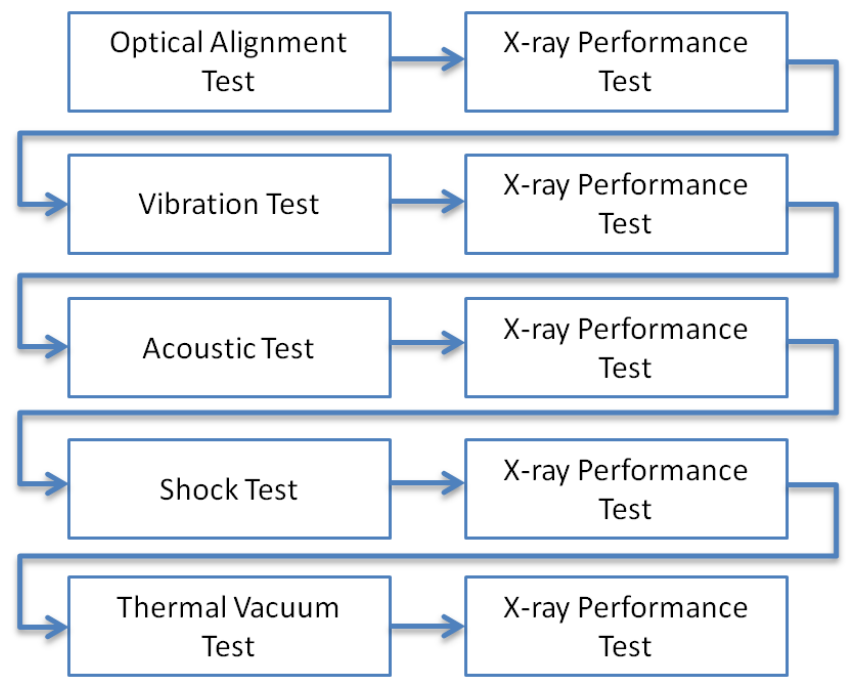

Figure 4. Preliminary test flow to verify module generic requirements

\section{Horizontal X-Ray Testing}

One requirement levied on modules by existing facilities is that the full aperture X-ray verification testing must be performed with the optical axis horizontal. The existing test facilities consist of a 100-600 meter long evacuated tube with an X-ray source at one end, and the optics and detector at the other. The self-weight distortion of the mirror module structure and the thin glass segments within is a major design driver. Figure 5 illustrates the angular resolution degradation caused by self-weight distortion of a fully populated module when held with the optical axis horizontal. The data is based on an optomechanical simulation of a kinematically supported module using Finite Element Analysis (FEA) and ray tracing. The nominal elastic modulus of the Kovar material used for the structure is $138 \mathrm{GPa}$. To reduce the self-weight distortion such that it does not dominate the module HPD would require a 1000 fold increase in structure stiffness. It may be possible to significantly increase the stiffness through improved structure design or addition of a g-negating test structure, but further analysis is needed.

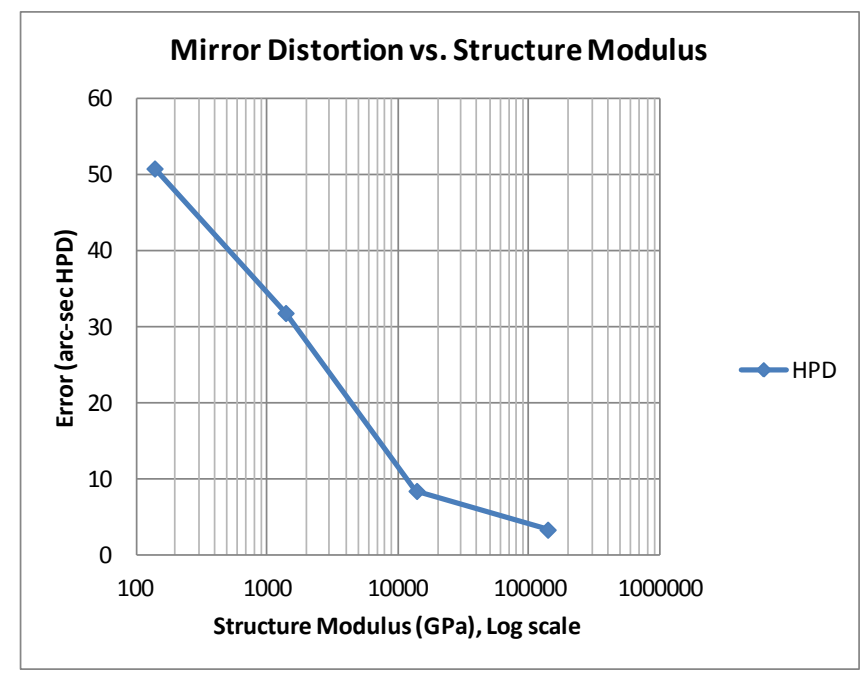

Figure 5. Optical performance of a conceptual AXSIO mirror module with the optical axis horizontal, simulating $X$-ray testing

Even with an infinitely rigid module structure the HPD is still limited to $\sim 3$ arc-sec due to the deformation of the thin glass segments. To achieve acceptable horizontal performance, six mounting locations between the mirror and module are required which over-constrains the mirror, necessitating sub-micron accuracy and stability of the mirror-to-module bonds. Essentially, the horizontal testing requirement drives the most challenging aspect of the technology [1]. Figure 6 shows the deformed shape of a mirror segment bonded at six locations and Figure 7 shows the sensitivity of horizontal mirror performance to the number of bond locations. The possibility of building a vertical X-ray test facility which relieves these issues and also opens up the possibility to kinematically mount the mirror segment is discussed in the next section. 


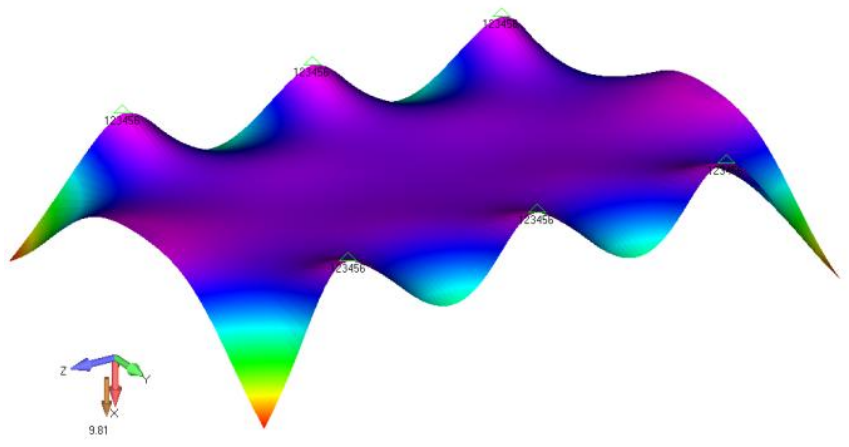

Figure 6. Deformed shape of a rigidly constrained horizontal mirror with six module mounting locations

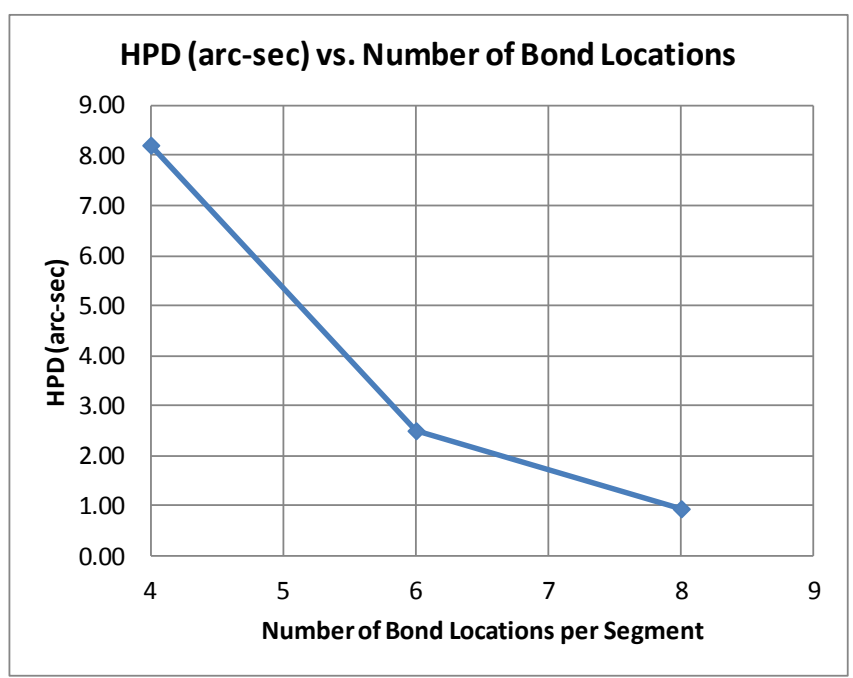

Figure 7. Figure error of a rigidly constrained mirror pair with 4,6 , and 8 mounting locations

\section{Kinematic Mounting OF Mirror SEGMENTS}

When designing a precision optical mount, a 6 Degree of Freedom (DOF) kinematic mount is generally preferred because it minimizes mirror distortion due to mounting by relieving stresses at the mounting locations. Since the mirror is only constrained in the six DOFs required to prevent rigid body motion, displacements at the mounting interface only cause alignment changes and not mirror figure changes. For X-ray mirror segments mounted into a module, kinematic mounting provides significant advantages over the current over-constrained mount, potentially leading to higher performance, reduced complexity, simplified analysis, and relaxed thermal requirements. However, kinematic mounting also creates new challenges such as kinematic mechanism design, launch-locking, and the need for vertical X-ray testing. Below, both the advantages and challenges of kinematically mounting slumped glass mirror segments into modules are explored in detail, along with concepts for implementation and preliminary results from prototype testing. Figures 8 and 9 illustrate the differences between a kinematic and over-constraint mount.

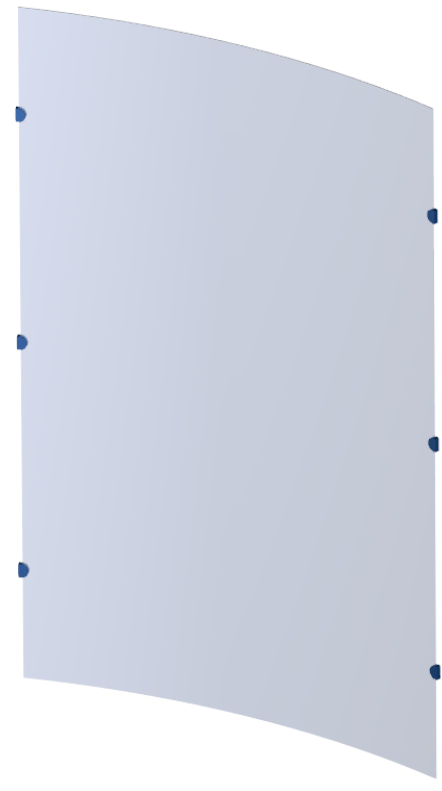

Figure 8. Over-constrained mirror with 6 mounting locations constraining 6 DOF each

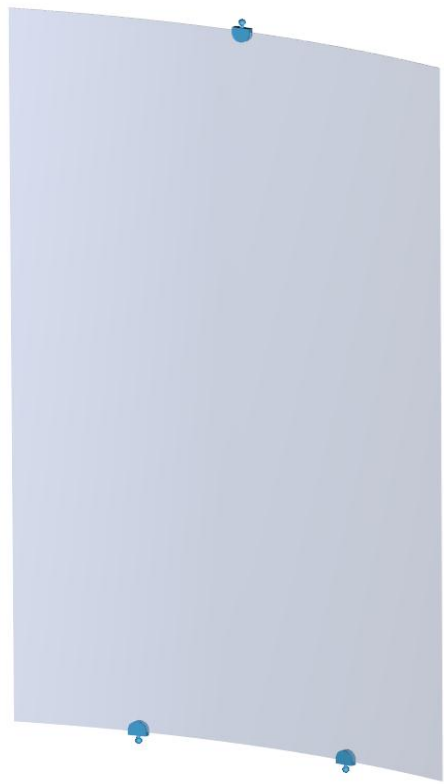

Figure 9. Kinematically constrained mirror with 3 mounting locations constraining 2 DOF each

\section{Advantages of Kinematically Mounting Mirror Segments}

In any mounting scheme, the mirror must be mechanically attached to the module structure. The primary advantage of kinematic mounting is that displacements at the mounting interface locations only cause mirror alignment changes, while displacements cause both mirror alignment and mirror figure changes when the mirror is over-constrained. Figure errors can be orders of magnitude more sensitive than alignment errors to mount displacements. For instance, a 1 $\mu \mathrm{m}$ axial displacement at a kinematic constraint causes 1.7 arc-sec alignment error while a $1 \mu \mathrm{m}$ axial displacement at an over-constrained location causes 35.7 arc-sec of figure 
error. Also, for the current over-constrained mount, the figure and alignment error is sensitive to mount displacements in each of the 36 DOFs constrained (6 DOF at 6 locations) while for kinematically mounted segments only mount displacements at 6 DOFs cause error, and then only alignment error. Furthermore, with 6 independent DOFs, the mounting errors can be easily measured and controlled while the coupled effects of 36 DOFs are difficult, if not impossible, to measure and control.

Kinematic mounting of the mirrors reduces mounting precision requirements and thus may offer significant improvement in optical performance and reduction in module integration complexity. Easing of the sensitivity to mount displacements is the primary advantage of kinematically mounting mirror segments into modules. Currently, adhesive is used to over-constrain the mirror at multiple mount locations by filling the gap between the precise mirror segment surface and the relatively imprecise module structure. This approach has proven extremely challenging since sub-micron accuracy and stability are required at each bond point in multiple DOFs. Hydraulic effects while applying the adhesive, cure shrinkage effects after adhesive application, and viscoelastic creep effects throughout the module life-cycle must all be carefully controlled. While successful at the $\sim 10$ arc-sec level, the over-constrained mounting approach is currently the limiting factor in Technology Development Module performance as mirror segments pair performance is predicted to be $\sim 6$ arc-sec before mounting. Indeed the over-constrained bonding process has been recognized as the most significant technological challenge. To reach the long-term goal of matching Chandra's resolution with lightweight optics, the mirrors may need to be kinematically mounted, as they are on Chandra.

Kinematic mounts are also called statically determinate mounts since the reaction forces at the mounting locations can be determined by the equations of static equilibrium and are not dependent on the deformation of the mirror or mount. The deformations of the module and mirror are thus fundamentally decoupled, resulting in several advantages. First, deformations of the module structure due to mechanical loads, such as launch loads, do not generate stress in the mirror. It was previously shown that module deformations drive $\sim 90 \%$ of the stress in the mirrors for the over-constrained mount. Second, thermal-elastic deformation of the module does not generate stress, and thus figure distortion, in the mirrors. This alleviates the need to use precisely CTE matched materials, which in this case are not otherwise desirable structural materials, and relieves the stringent requirement of $0.1^{\circ} \mathrm{C}$ maximum thermal gradient between the mirrors and module structure. Finally, the structural decoupling allows the modules and segments to be analyzed and tested independently, greatly simplifying verification of structural modes and mechanical strength.
Implementation Challenges and Solutions for Kinematically Mounting Mirror Segments

Kinematic mounts, which constrain some degrees of freedom while allowing motion in others, are primarily achieved using either kinematic flexure systems or kinematic mechanism systems [10]. Any kinematic system is necessarily imperfect, either with respect to rigidly constraining the intended DOFs or allowing truly free motion to occur in the unconstrained DOFs. Flexure systems, usually consisting of beams which are stiff in the constrained DOFs but flexible in the unconstrained DOFs, must strike a balance between rigidity, free motion, strength, and volume. Since the same beam must allow both constraint and freedom in various DOFs flexure design optimization must be employed to balance the competing requirements of stiffness and compliance. Flexures also require significant volume since the compliance of a beam increases exponentially with its length. For implementation into a mirror module, where the space between mirrors is $\sim 2$ $\mathrm{mm}$, an acceptable flexure design solution has not yet been found.

In comparison, kinematic mechanism systems can have excellent stiffness in the constrained DOFs, excellent freedom of motion in the unconstrained DOFs, and can be made very compact. However, this comes at the price of requiring clearances in the mechanism to allow free motion and a nesting force to ensure contact, and thus constraint, is maintained. Clearances can cause dynamic amplification of input vibration loads (colloquially call the 'rattle-gap' effect) due to kinetic energy developing during free motion then releasing when contact reoccurs. This can be mitigated by reducing the clearances as much as possible through precision fabrication of the mechanisms. Practically, clearances of several microns can be achieved with precision machining, though at significant cost relative to standard machining tolerances. Testing must be performed to determine the optimum clearance which suitably minimizes dynamic amplification at an acceptable cost.

In order to ensure the desired constraint occurs in the kinematic mechanism, a nesting force is required to hold the components in contact. On the ground, gravity readily supplies this nesting force. Currently, kinematic mechanisms are used to support the mirror segment during metrology to verify the as-fabricated figure and also to support the segment during alignment and integration into the module structure. Figure 10 shows the kinematic mount currently used. The kinematic approach has proven to be the best, simplest, and most reliable method for measuring, aligning, and integrating mirrors. However, the module must eventually operate in the micro-gravity on-orbit environment. To supply the nesting forces which ensure the mirrors are held in their aligned locations, small magnets or springs may be used. The strength of the nesting force must be traded against the contact friction it creates in order to ensure the mechanism still moves sufficiently freely in the unconstrained directions. For the 25 gram mirrors, a very 
small nesting force is required to hold the mechanism in contact on-orbit, creating minimal friction. Optomechanical analysis has shown that a 1.0 millinewton $(\mathrm{mN})$ friction force can be tolerated without causing more than 0.5 arc-sec of mirror distortion.

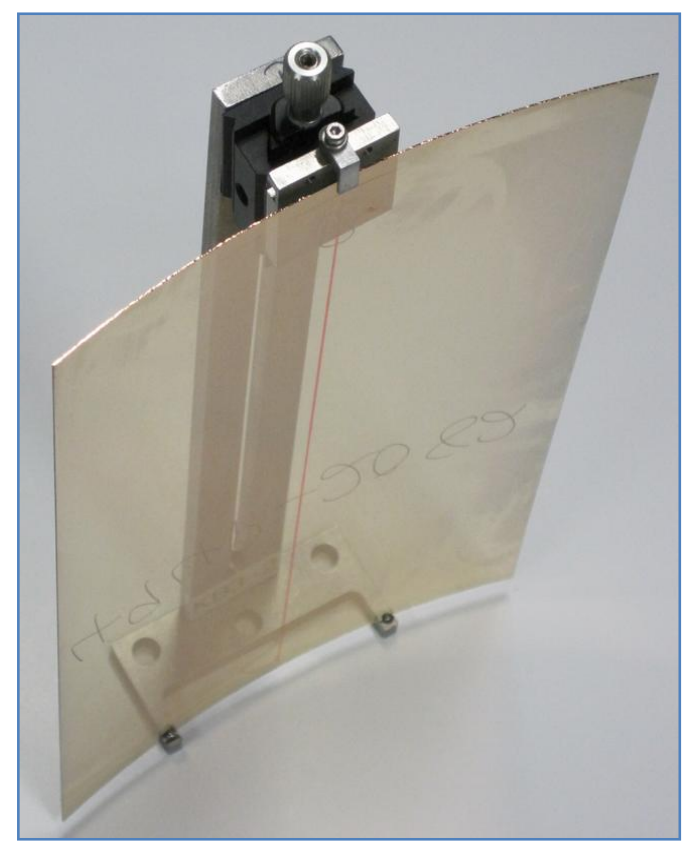

Figure 10. Kinematic mount currently used during metrology and alignment.

Another issue with implementing kinematic constraint is the lower vibration modes of the mirror segment relative to the over-constrained design, with FEA predicting fundamental frequencies of $27 \mathrm{~Hz}$ vs. 257 respectively as shown in Figure 11. The low vibration modes of the kinematically supported mirror segment lead to large displacements during launch vibrations and subsequent impact between adjacent segments, clearly an unacceptable situation. Aside from attempting to stiffen the mirror segment through material or size changes, which have far reaching consequences for technology development, a simple solution is to apply temporary constraints during launch which are later released after the detrimental loading environment is over. This approach is achieved through mechanisms commonly called launch-locks. The addition of two single DOF constraints along the axial edge of the mirror raises the fundamental frequency to an acceptable level of $130 \mathrm{~Hz}$. A simple prototype system which adds retractable launch-locks to the alignment fixture shown in Figure 10 has been used to demonstrate this effect, and also to verify the optical alignment returns after the launch-locks are removed, thereby successfully simulating initial alignment, application of launch-locks during launch, then removal onorbit.
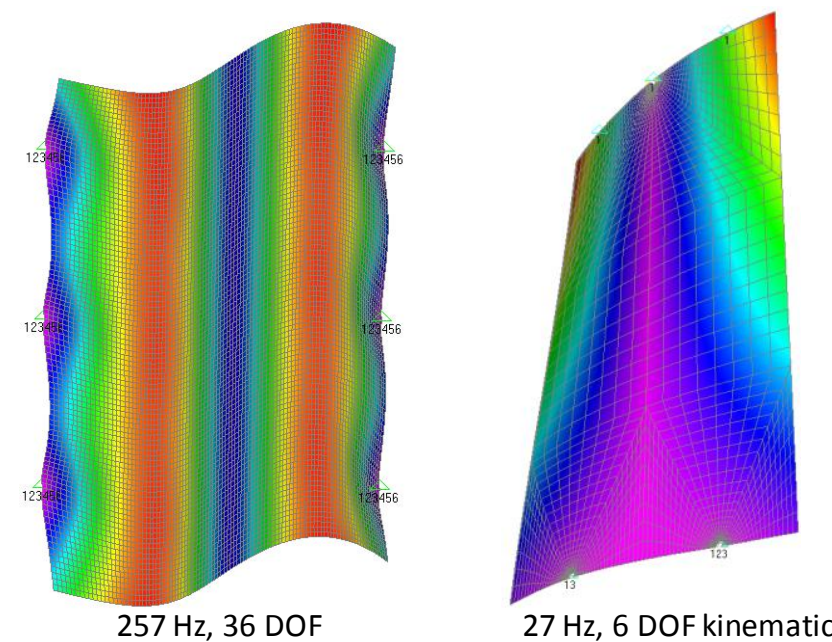

Figure 11. Fundamental frequencies of an over-constrained mirror (left), a kinematically constraint mirror (right)

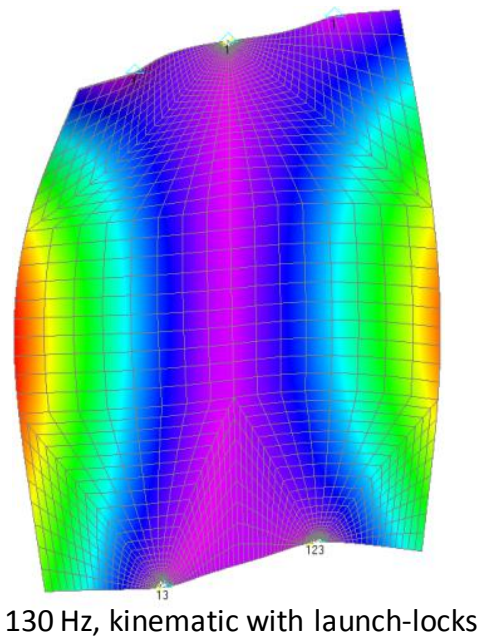

Figure 12. Fundamental frequency of a kinematically constrained mirror with launch-locks engaged.

The most significant barrier preventing implementation of kinematic mounting of slumped glass mirror segments is the horizontal X-ray test requirement described in Section 2.5 above. While self-weight figure distortion of a kinematically mounted mirror with the optical axis vertical is only $\sim 1$ arc-sec, with the optical axis horizontal the error is over 100 arc-sec. As shown in Figure 7 the current gross over-constraint of the segment is driven by the horizontal test requirement. While confidence in the mirror figure and alignment can be obtained in visible wavelengths by a combination of normal incidence interferometric metrology and grazing incidence Hartmann metrology, true verification of on-orbit performance may require full aperture X-ray testing in the relevant wavelengths. Options for vertical Xray testing are addressed in the following section.

\section{Options for a Vertical X-Ray Test Facility}

The most straight-forward option for building a vertical Xray test facility, from a performance perspective, would be to place an X-ray source several hundred meters 
underground at the bottom of an evacuated tube. The optics could be located in a ground-level building and the detector could be located at the top of a $\sim 10$ meter tower as shown in Figure 13. Excavations of sufficient diameter and depth are routinely drilled and steel-clad to access ground water, petroleum, and mineral resources, taking as little as a week to dig and costing $<\$ 100 \mathrm{~K}$. The most unique requirement would be to ensure the tube is vacuum tight as the cladding is installed. An existing facility, the Zero Gravity Research Facility at NASA Glenn, has a $150 \mathrm{~m}$ deep evacuated tube 6 $\mathrm{m}$ in diameter, demonstrating feasibility. However, a new facility dedicated to X-ray testing is only likely to receive sufficient funding once a flight project is started, rather than during technology development.

In order to bootstrap the kinematic mounting technology to a TRL sufficient for mission implementation, a less expensive facility could be built utilizing a soft X-ray source, in the $0.1 \mathrm{keV}$ range, which is collimated by a parabolic mirror with a multilayer coating before being focused by the modules as shown in Figure 14. Such a facility would be $\sim 12$ meters long, fitting within several existing high-bay areas at GSFC.

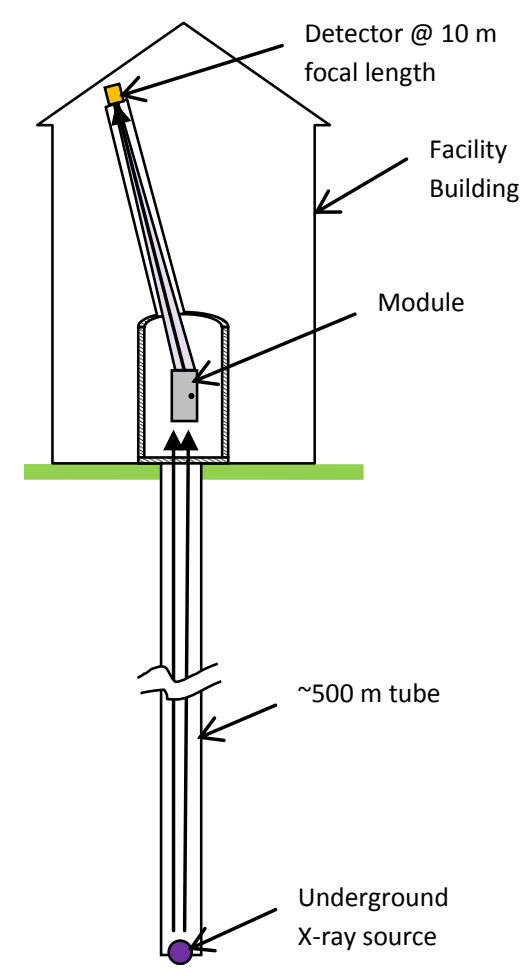

Figure 13. Conceptual design of a deep excavation X-ray test facility.

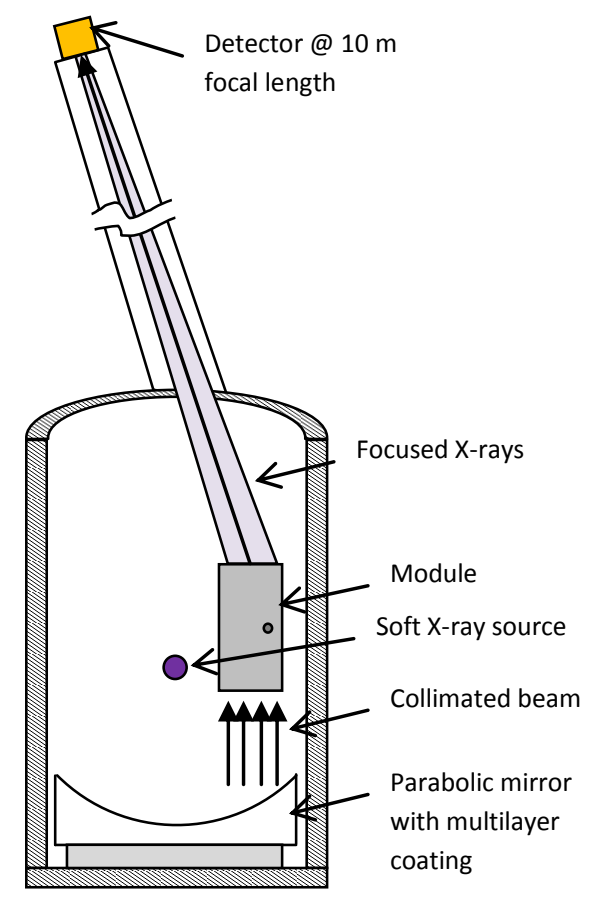

Figure 14. Conceptual design of a soft X-ray test facility.

Other options for preliminary testing of kinematically mounted mirror segments include sounding rocket missions, sub-orbital Reusable Launch Vehicle (RLV) flights, and reduced gravity aircraft flights. However, a soft X-ray facility may provide the best combination of low cost and ready access.

\section{Conclusions}

As the slumped glass mirror technology being developed at NASA GSFC nears the performance requirements for mission implementation, increased effort is being invested to ensure the module design is compatible with spaceflight. Driving requirements have been identified, generic requirements based on several study missions have been developed, and a verification test flow has been drafted. The NGXO team plans to mitigate the cost and schedule risk of mission implementation by rigorously and repeatedly integrating and testing Technology Development Modules.

Fundamental limits on the resolution of the thin mirror segments are imposed by the requirement for horizontal Xray testing. When kinematically constrained, mirror segments distort to the point of untestability. Even when the segments are over-constrained, distortion of a fully populated module is unacceptably large, requiring stiffening of the structure by a factor of $\sim 1000$, the feasibility of which has yet to be demonstrated.

The need to over-constrain the mirror segments present additional challenges relative to bonding the mirrors into the modules and designing the modules for optical performance. These issues can be alleviated through the use of kinematic mounting mechanisms between the module and mirror segments. Prototype kinematic mounts have been 
developed and tested demonstrating the ability to overcome issues such as mechanism clearances, on-orbit nesting forces, and reduced mirror structure modes. Finally, options for vertical $\mathrm{X}$-ray testing facilities needed to test kinematically constrained mirrors have been explored.

\section{REFERENCES}

[1] Zhang, W. W., et al., "Lightweight and high angular resolution x-ray optics for astronomy", Proc. SPIE 8076, 807602 (2011).

[2] Zhang, W. W., et al., "Next generation x-ray optics: highresolution, light-weight, and low-cost", Proc. SPIE 844328, (2012).

[3] Zhang, W. W., et al., "Mirror technology development for the International X-ray Observatory mission (IXO)", Proc. SPIE 7732, $77321 \mathrm{G}$ (2010).

[4] McClelland, R. S., et al., "Design and analysis of the International X-Ray Observatory mirror modules ", Proc. SPIE 7732, (2010).

[5] Petre, R., et al., "The NASA x-ray mission concepts study", Proc. SPIE 8443-54, (2012).

[6] Robinson, D. W., et al., "Mechanical overview of the International X-ray Observatory", IEEE Aerospace conference, (2009).

[7] Bookbinder, J. A., et al., "The Advanced X-ray Spectroscopic Imaging Observatory (AXSIO)" Proc. Of SPIE Vol. 8443-43, (2012).

[8] Sarafin, T. P. and Larson, W. J., "Spacecraft Structures and Mechanisms: From Concept to Launch", Springer, (1995).

[9] McClelland, R. S., et al., "Design and analysis of mirror modules for IXO and beyond", Proc. SPIE 8147, 814700 (2011).

[10] Blanding, D. L., "Exact Constraint: Machine Design Using Kinematic Principles", American Society of Mechanical Engineers, (1999).

\section{BIOGRAPHIES}

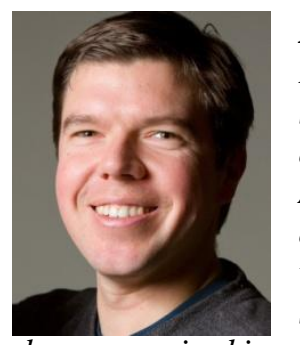

Ryan McClelland is a Senior Mechanical Systems Engineer at SGT Inc. currently leading the design of the IXO Flight Mirror Assembly. His previous technology development experience includes work on aluminum foam core optical systems and non-linear effects of clearances in kinematic mechanisms. Ryan has also worked on flight missions with designs currently on orbit aboard the Hubble Space Telescope and Space Technology 5 spacecraft. He received a B.S in Mechanical Engineering, summa cum laude, from the University of Maryland. 